scanning.

The output from the pulse height analyser of the scanner is stored in each address as the counts during $1.5 \mathrm{~mm}$ of scanning movement of the detector. During the spacing movement of the detector, one line of data just stored in the core memory are transfered to the magnetic tape unit and stored in it as unprocessed primary data.

On processing, these data are compressed into a $64 \times 64$ matrix as the counts of $3 \times 3 \mathrm{~mm}$ or $3 \times 4.5 \mathrm{~mm}$ elements. Storage of unprocessed primary data enables us to interprete them with optimal processing later on, by trial and error, and shows a definite advantage especially in long term follow up of one case.

Gamma camera data are accumulated in corresponding address to their $\mathrm{X}$ and $\mathrm{Y}$ position signals. Since the transference of one picture to the magnetic tape and the clearance of the core memory require about a quarter second, the dynamic study of the radioangiography is not continuous, but fairly practical for the estimation of the abnormal cerebral circulation.

For the detection of very faint abnormal uptake observed in CVA cases, the bilateral collation method was developed.

Although the improvement of the data processing might be of secondary importance comparing with that of the data collecting (improvement of measuring instruments, radionuclides and administration of them, etc.), this project must be valuable in relation with the automatic diagnosis in future.

\title{
H-8. Myelography and Extradurography of the Spinal Canal with Water Soluble Contrast Medium
}

\author{
Toru Yoshida, Susumu Kato \\ Hiroshi Sugrura and Kentaro Mimatsu \\ Orthopedic Service, Kariya-Toyota Hospital
}

\section{H-9. Endoscopic Diagnosis of Ventricular Lesions by Newly Designed Ventriculofiberscope}

\author{
Takanori Fukushima, Norio Yoshrmasu, Buichi Ishijima \\ Kimiyoshi Hirakawa and Keiji Sano \\ Department of Neurosurgery, Tokyo Police Hospital \\ Department of Neurosurgery, University of Tokyo
}

Since the first description of the ventriculoscope by Walter E. Dandy in 1972, various kinds of ventriculoscopes have been reported by Fay, Putnam, Guiot, Scarff etc. Most of them were utilized for the endoscopic coagulation of the choroid plexuses 
in the lateral ventricle as the treatment of hydrocephalus. Up to this time, however, these endoscopes have not been popular in neurosurgical fields because of their restricted clinical use. They were rigid and of restricted viewfield as well as large in diameter.

The authors have devised a new "Ventriculofiberscope" and proved its great values through the animal experiments and clinical applications. Its advantages are as follows: 1) $0.4 \mathrm{~cm}$ in diameter, 2) with wide angle of view fields of $68^{\circ}, 3$ ) smooth and flexible with the motor-driven bending angle of $30-130^{\circ}$, 4) having a channel for irrigation, suction, coagulation, and biopsy probe, 5) equipped with a supporting system.

So far, the authors have tried our ventriculofiberscope in 37 cases. It enabled us to observe inner wall of the lateral and the 3rd ventricles with vivid image, especially it was excellent for direct observation of the posterior half of the 3rd ventricle. Moreover, some endoscopic operations such as biopsy, perforation of the septum pellucidum, 3rd ventriculostomy, and puncture of the cystic tumor were possible through the channel within the scope. Endoscopic biopsy of the ventricular tumors were performed in 11 cases, 6 of which were confirmed histologically before craniotomy. Our experiences were reported in the $16 \mathrm{~mm}$ color movie.

\title{
H-10. Pupillary Reflex Perimeter
}

\author{
Naomi Mutsuga, Kenichiro Sugita \\ Tatsuo Banno and Tetsuro MizuTani \\ Department of Neurosurgery, Nagoya Universtly
}

1) We developed a perimeter by measuring the pupillary reflex using an infra-red television system, which requires neither oral nor tap answers of the patients.

2) With this device measurements of the visual field of children and uncosscious patients can be obtained under light anesthesia and animals as well in the supine position.

3) Fifty cases with defects in the visual field and with normal visual field were tested. The results obtained by using this method were identical with those observed with the ordinary quantitative perimenter of Goldmann.

4) In the cases of homonymous hemianopsia which were caused by the lesion of the occipital and temporo-parietal area, the results of this method showed same homonymous hemianopsia.

5) The internal cortico-tectal tract, the tectooculomotor tract and the caudal Edinger-Westphal nuclei, which is accepted to be a fiber pathway to cause myosis associated with accommodation, may be also a probable pathway involved in the pupillary light reflex. 